\title{
Ceruloplasmin, a potential marker for glycemic status and its relationship with lipid profile in Type II diabetes mellitus
}

\author{
Vijay Kumar Sharma ${ }^{1}$, Arjun Tumbapo ${ }^{2}$, Vivek Pant ${ }^{3}$, Binod Aryal ${ }^{4}$, \\ Sweta Shrestha ${ }^{5}$, Binod Kumar Yadav ${ }^{6}$, Eans Tara Tuladhar ${ }^{7}$, Aseem Bhattarai ${ }^{8}$, \\ Mithileshwor Raut ${ }^{9}$ \\ ${ }^{1,7,8,9}$ Assistant Professor, ${ }^{2,3}$ Resident Doctor, ${ }^{4,5}$ Masters Student, ${ }^{6}$ Associate Professor, Department of Biochemistry, \\ Institute of Medicine (IOM), Tribhuvan University Teaching hospital(TUTH)
}

A B S T R A C T

Background: Type II Diabetes Mellitus (Type II DM) is a metabolic disorder characterized by glucotoxicity and lipotoxicity. Markers of glycemic control are $\mathrm{HbA1c}$, fasting blood glucose and postprandial blood glucose. There is altered lipid parameters in Type II DM which possess significant cardiovascular risk to the patient. Recent studies have shown that ceruloplasmin, an inflammatory marker having antioxidant property, is ideal marker to know the cardiovascular status, degree of insulin resistance and cancer risk. Aims and Objectives: Present study was carried out to determine the relation between glycemic status and serum ceruloplasmin along with lipid parameters which reflects the cardiovascular status in these patients. Materials and Methods: Blood samples were collected from eighty-eight patients with type II diabetes mellitus along with age and sex matched forty-two healthy controls. Fasting lipid profile and blood glucose, postprandial blood glucose, serum ceruloplasmin and $\mathrm{HbA} 1 \mathrm{c}$ levels were determined using auto analyser in the department of clinical biochemistry in Institute of Medicine.Appropriate tests of significance were computed by SPSS version 20. Results: Serum ceruloplasmin was found higher in type II DM than nondiabetic group, median 52.0 (95\% Cl: 49-53) mg/dl versus median 45.0 (95\% Cl: 41-47) $\mathrm{mg} / \mathrm{dl}$, at significant level of 0.001 probability test. Similarly, fasting blood glucose, post prandial glucose, $\mathrm{HbA} 1 \mathrm{c}$ and $\mathrm{TG} / \mathrm{HDL}$ cholesterol ratio correlated significantly with serum ceruloplasmin. The cut off value of $46.5 \mathrm{mg} / \mathrm{dL}$ was obtained for serum ceruloplasmin with sensitivity of $87.5 \%$ and specificity of $62 \%$ which has good discriminating value for type II diabetic patients versus non-diabetic patients. Conclusion: Findings in our study shows that increased glycation coupled with altered lipid profile in type II DM causes enhanced generation of ceruloplasmin which could be used as potential marker for identifying acceleratedglycation and atherogenesis in these subjects.

Key words: Type II DM, Ceruloplasmin, Lipid profile, Lipid ratio

\section{Access this article online}

Website:

http://nepjol.info/index.php/AJMS DOI: 10.3126/ajms.v9i2.19003 E-ISSN: 2091-0576

P-ISSN: 2467-9100

\section{INTRODUCTION}

Type II diabetes mellitus (Type II DM) is a chronic progressive disease primarily characterized by hyperglycemia and dyslipidemia that later leads to cardiovascular complications. Other complications affecting kidney, eye and nervous system can also occur. Hyperglycemia impairs glucose metabolic pathways andincreases auto-oxidative glycosylation and free radical production. Deranged lipid status favors free radical-induced lipid peroxidation and leads to deposition of oxidizedlow-density lipoprotein (LDL) cholesterol resulting in atherosclerosis of blood vessels. Hyperglycemia in long run causes toxic effects on macro as well as microvascular structure and affects proper functioning of organs. Glucose toxicity which 
includes plethora of biochemical process such as glucose autoxidation, protein kinase $\mathrm{C}$ activation, methylglyoxal formation and glycation, hexosamine metabolism, sorbitol formation, and oxidative phosphorylation, is held responsible for the damage of tissues. ${ }^{2}$ Thus, there is important role of inflammation in diabetes mellitus. It has been proved that low grade inflammation is associated with diabetes mellitus in various intervention studies. ${ }^{3}$ Hyperglycemia directly influences malignant cells proliferation as well as indirectly influences cancer cells through an increase in the levels of IGF-1 (insulin like growth factor-1). ${ }^{4}$ It is evident that solid cancers such as liver, pancreatic cancers colorectal, breast, endometrial and renal cancer have high prevalence in diabetes mellitus.

Among various inflammatory markers ceruloplasmin, a copper-carrying metalloenzyme, which acts as an antioxidant through its ferroxidase activity is the ideal candidate to know the overall cardiovascular, insulin resistance and cancer risk in patients with type II DM.In conditions of elevated oxidative stress, ceruloplasmin may act as a pro-oxidant by donating of free copper ions, which induces reactive oxygen species (ROS) formation and low-density lipoprotein (LDL) oxidation. ${ }^{5}$ In addition, as an acute phase protein, the ceruloplasmin level reflects acute and chronic inflammation in an organism. ${ }^{6}$ Ceruloplasmin is the candidate marker in diabetes mellitus since the prevalence of solid tumors are high and various studies have found significant rise in the level of serum ceruloplasmin in patients with locally advanced or regionally spreading tumors. ${ }^{7}$

The present study is designed to know the status of the serum ceruloplasmin in the type II diabetes mellitus and to study the correlation of the ceruloplasmin with glycemic status and lipid profile. This study tries to find the association of ceruloplasmin with lipid ratio which reflects the cardiovascular status.

\section{MATERIALS AND METHODS}

Blood samples were collected from eighty-eight patients with type II diabetes mellitus along with age and sex matched forty-two healthy controls. Diagnosis of type II DM patients was done according to WHO criteria.Type II DM patients with complications like ischemic heart disease, diabetic nephropathy, diabetic retinopathy and other microvascular complications or infectious diseases at the time of sampling were excluded from the study group. Patients were either on insulin therapy or oral hypoglycemic agents. The healthy controls were not on any medication and were free from any acute or chronic illness. Informed consent was obtained from all subjects involved in the study. Ethical clearance for this study was approved by institutionalresearch board of Institute of Medicine.

Fasting lipid profile and blood glucose, postprandial blood glucose, serum ceruloplasmin and HbA1c levels were determined using auto analyser in the department of clinical biochemistry in Institute of Medicine. Total Cholesterol(TC) was measured by cholesterol oxidase-phenol amino phenazone (CHOD PAP) method,triglyceride(TG) by glycerol-3-phosphate oxidase phenol amino phenazone (GPO-PAP) method,high density lipoprotein (HDL) cholesterol by direct method andlow density lipoprotein (LDL) cholesterol calculated by Friedewald Equation.Similarly, serum glucose was measured by glucose oxidase peroxidase (GOD POD) method and ceruloplasmin by immunoturbidimetric method in BT3500 biochemistryautoanalyser. HbA1c was measured using high performance liquid chromatography (HPLC) method in ERBA Mannheim's Hb-Vario.

\section{Statistical Analysis}

Statistical analysis was performed using Statistical Package for the Social Sciences, SPSS 17. Results are expressed as mean $\pm \mathrm{SD}$ as well as median (confidence interval) according to distribution of the data. Pearson's correlation was applied to correlate between the parameters. A p-value less than 0.05 (2-tailed) was considered statistically significant. For non-normaldistribution,non-parametric test is used.

\section{RESULTS}

Total number of male participants were $53.1 \%$ (Type II DM 47 and nondiabetic 22) andtotal female participant were $46.9 \%$ (Type II Diabetic mellitus 41 and nondiabetic 20).

Ceruloplasmin was found higher in type II diabetic patients than non-diabetic patients, median 52.0 (95\% CI 49-53) $\mathrm{mg} / \mathrm{dL}$ versus median 45.0 (95\% CI $41-47 \mathrm{mg} / \mathrm{dL}$ which was significant at level of 0.001 probability level (Table 1) shows the descriptive statistics for type II diabetic patients versusnon-diabetic participants.

A series of Spearman rank-order correlations were conducted to determine if there were any relationship between ceruloplasmin and other variables. A two- tailed test of significance was used to find the significant relationship.Correlations among variables were conducted in two groups (Table 2)as follow:

1. Overall which consisted of type II diabetic mellitus patients and non-diabetic participant

2. Type II diabetic mellitus patients 


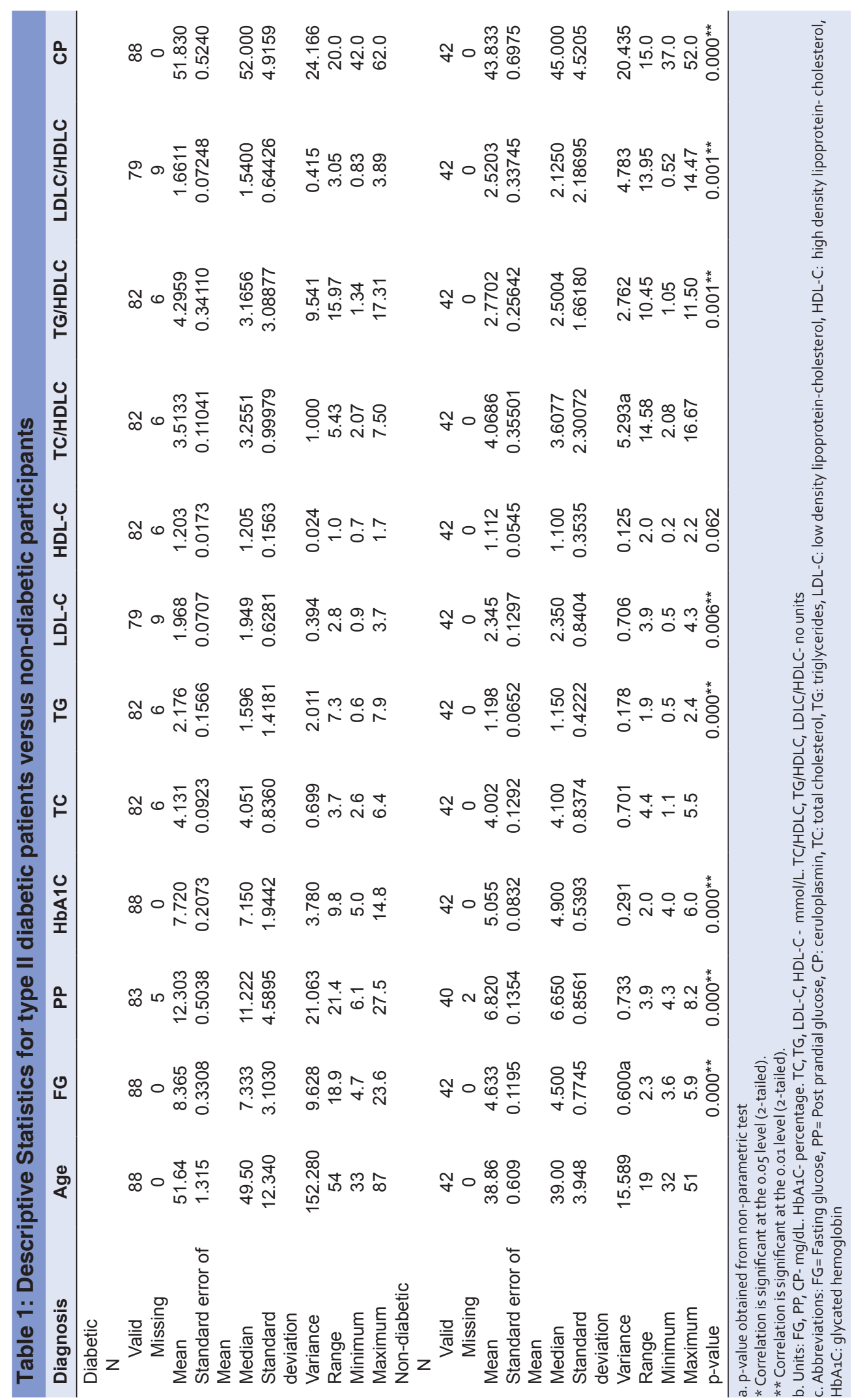


Overall which consisted of type II diabetic mellitus patients and non-diabetic participants:

Correlation of ceruloplasmin was computed with 11 variables. Among them age, fasting serum glucose, post prandial glucose, HbA1C, triglycerides and TG/HDL-C ratio correlated significantly with ceruloplasmin at 0.001 probability level.

Type II diabetic mellitus patients:

Correlation of ceruloplasmin was computed with 11 variables. Among them HbA1C, LDL-C, TC/HDL-C ratio and LDL-C/HDL-C ratio correlated significantly with ceruloplasmin at 0.001 probability level.

Correlation of ceruloplasmin with study's variables according to gender difference in type II diabetic mellitus

In type II diabetic female patients, ceruloplasmin was significantly correlated with TG/HDL-C ratio, r 372 , p-value.025 (p-value $<0.05,2$-tailed) (Table 3).

ROC curve was plotted using ceruloplasmin as test variable and diabetic/non-diabetic as state variable. The area under the curve is 0.881 whose area is more than 0.5 . Thus, its usefulness for the overall ability of the test to discriminate between those individuals with the disease and those without the disease is justified.

The cut off value of $46.5 \mathrm{mg} / \mathrm{dL}$ was obtained for serum ceruloplasmin with sensitivity of $87.5 \%$ and specificity of $62 \%$ which has good discriminating value for type II diabetic patients versus non-diabetic patients (Figure 1).

\section{DISCUSSION}

Studies investigating the association between serum ceruloplasmin and development of diabetes and its

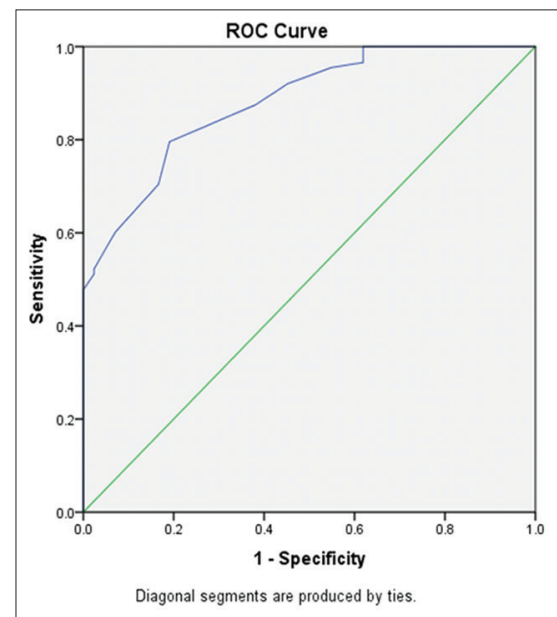

Figure 1: ROC curve for ceruloplasmin to differentiate Type II DM from nondiabetic complication has been extensively studied in recent years. The present study was conducted to know the status of ceruloplasmin in diabetic and nondiabetic individuals and correlate its concentration with lipid ratio and blood glucose profile.

Ceruloplasmin was found to be higher in diabetic group than non-diabetic group. Similar findings have found in various studies conducted in by Jung Lee M et al 2015 in Korea ${ }^{5}$ and Nasif ZN et al 2010 in Iraq. ${ }^{8}$ The cut off value of serum ceruloplasmin for distinguishing diabetic and non-diabetic group was in our study $46.5 \mathrm{mg} / \mathrm{dL}$ which has sensitivity of $87.5 \%$ and specificity of $62 \%$.

Similarly, serum glucose level was high in participants with high serum ceruloplasmin level. Overall, considering both groups; hyperglycemia correlates with serum ceruloplasmin. Surprisingly hyperglycemia does not correlate with serum ceruloplasmin in diabetic group. The probable explanation may be that ceruloplasmin increases in diabetic group but does not fluctuate considerably in the diabetic range. Similarly, considering both groups ceruloplasmin correlates with age, fasting glucose, post prandial glucose, glycated hemoglobin, triglycerides and TG/HDL-C ratio. Thus, it implies that serum triglycerides and TG/HDL-C ratio which are substitute marker of insulin resistance could be reflected by ceruloplasmin. Ceruloplasmin could be used as substitute to mark the insulin resistance.

In the diabetic group HbA1c iscorrelated with ceruloplasmin (Table 2). Thus, glycemic index correlates with inflammatory status, as ceruloplasmin indicates the inflammatory status of the body. Further, LDL-C, TC/ HDL-C ratio and LDL-C/HDL-C ratio correlates with ceruloplasmin (Table 2). The importance of this correlation is highlighted by the fact thatLDL-C is one of the key factor for development of atherosclerosis. ${ }^{9}$ Epidemiological data suggests that serum ceruloplasmin may be an important risk factor predicting myocardial infarction and cardiovascular disease. This is becauseceruloplasmin is a potent catalyst of LDL oxidation. ${ }^{10}$ Several studies have observed that LDL-C/HDL-C ratio predicts carotid intima-media thickness progression and it predicts better than other lipid ratios. ${ }^{9}$ Similarly, TC/HDL-C ratio variation is associated with more substantial alterations in metabolic indices predictive of ischemic heart disease risk and related to the insulin resistance syndrome. ${ }^{11} \mathrm{TC} /$ HDL-C ratiois the substitute for metabolic index and its correlation with ceruloplasmin verifies the importance of serum ceruloplasmin in monitoring total cholesterol and manage accordingly.

In the diabetic group according to gender, lipid risk ratios have correlated differently with ceruloplasmin. Surprisingly 
Table 2: Correlation of ceruloplasmin with study's variables

\begin{tabular}{llllcccccccccc} 
& & & Age & FG & PP & HbA1C & TC & TG & HDL-C & LDL-C & TC/HDLC & TG/HDLC & LDLC/HDLC \\
\hline Overall: Diabetic & CP & $\mathrm{r}_{\mathrm{s}}$ & $0.297^{* *}$ & $0.535^{* *}$ & $0.621^{* *}$ & $0.667^{* *}$ & 0.146 & $0.322^{* *}$ & 0.069 & 0.002 & 0.061 & $0.240^{* *}$ & -0.03 \\
and Non-diabetic & & $\mathrm{p}-$ value & 0.001 & 0.000 & 0.000 & 0.000 & 0.106 & 0.000 & 0.449 & 0.981 & 0.498 & 0.007 & 0.747 \\
& & $\mathrm{~N}$ & 130 & 130 & 123 & 130 & 124 & 124 & 124 & 121 & 124 & 124 & 121 \\
Diabetic Type II & $\mathrm{CP}$ & $\mathrm{r}_{\mathrm{s}}$ & -0.012 & 0.03 & 0.207 & $0.347^{* *}$ & 0.206 & 0.113 & -0.006 & $0.268^{*}$ & $0.247^{*}$ & 0.133 & $0.279^{*}$ \\
& & $\mathrm{p}-$ value & 0.915 & 0.78 & 0.06 & 0.001 & 0.063 & 0.31 & 0.956 & 0.017 & 0.025 & 0.232 & 0.013 \\
& & $\mathrm{~N}$ & 88 & 88 & 83 & 88 & 82 & 82 & 82 & 79 & 82 & 82 & 79 \\
\hline
\end{tabular}

* Correlation is significant at the 0.05 level (2-tailed).

** Correlation is significant at the 0.01 level (2-tailed).

Unit- FG, PP, CP: mg/dL. HbA1C: percentage. TC, TG, LDL-C, HDL-C: mmol/L. TC/HDLC, TG/HDLC, LDLC/HDLC: no units.

Abbreviations: FG: fasting serum glucose, PP: post prandial glucose, CP: ceruloplasmin, TC: total cholesterol, TG: triglycerides, LDL-C: low density lipoprotein-cholesterol, $\mathrm{HDL}-\mathrm{C}$ : high density lipoprotein- cholesterol, $\mathrm{HbA} 1 \mathrm{C}$ : glycated hemoglobin.rs: Spearman rank correlation coefficient

\begin{tabular}{|c|c|c|c|c|c|c|c|c|c|c|c|c|}
\hline $\begin{array}{l}\text { Type II } \\
\text { diabetic } \\
\text { mellitus }\end{array}$ & & Age & FG & PP & HbA1C & TC & TG & HDL-C & LDL-C & TC/HDLC & TG/HDLC & LDLC/HDLC \\
\hline \multirow[t]{3}{*}{ CP (Female) } & $r_{s}$ & -0.08 & -0.135 & 0.173 & 0.26 & $0.423^{*}$ & $0.382^{*}$ & 0.077 & 0.247 & $0.342^{*}$ & $0.372^{*}$ & 0.223 \\
\hline & $\frac{\mathrm{s}}{\mathrm{p} \text {-value }}$ & 0.618 & 0.399 & 0.287 & 0.101 & 0.01 & 0.021 & 0.656 & 0.153 & 0.041 & 0.025 & 0.197 \\
\hline & $\mathrm{N}$ & 41 & 41 & 40 & 41 & 36 & 36 & 36 & 35 & 36 & 36 & 35 \\
\hline \multirow[t]{3}{*}{ CP (Male) } & $r_{s}$ & 0.059 & 0.088 & 0.271 & $0.425^{\star \star}$ & 0.096 & -0.065 & -0.167 & 0.289 & 0.166 & -0.025 & $0.320^{*}$ \\
\hline & p-value & 0.696 & 0.556 & 0.079 & 0.003 & 0.524 & 0.669 & 0.266 & 0.058 & 0.27 & 0.868 & 0.034 \\
\hline & $\mathrm{N}$ & 47 & 47 & 43 & 47 & 46 & 46 & 46 & 44 & 46 & 46 & 44 \\
\hline
\end{tabular}

* Correlation is significant at the 0.05 level (2-tailed).

** Correlation is significant at the 0.01 level (2-tailed).

Unit - FG, PP, CP: mg/dL. HbA1C: percentage. TC, TG, LDL-C, HDL-C: mmol/L. TC/HDLC, TG/HDLC, LDLC/HDLC: no units.

Abbreviations: FG: fasting serum glucose, PP: post prandial glucose, CP: ceruloplasmin, TC: total cholesterol, TG: triglycerides, LDL-C: low density lipoprotein-cholesterol, $\mathrm{HDL}-\mathrm{C}$ : high density lipoprotein- cholesterol, $\mathrm{HB}_{1} \mathrm{C}$ : glycated hemoglobin.r. $\mathrm{r}_{\mathrm{s}}$ : Spearman rank correlation coefficient

TC, TG and TG/HDL-C correlation with ceruloplasmin is seen in female diabetic group. For male in diabetic group $\mathrm{HbA} 1 \mathrm{C}$ and LDL-C/HDL-C ratio correlated significantly with ceruloplasmin. The probable reason may be due to that the cut off for TG/HDL-C ratio is lower for female. The clinical implication may be that, the female population should get benefit by controlling their lipid level. We could assume that the response of inflammatory status differs between male and female in the diabetic group. It also indicates our approach and priorities during treatment of hyperlipidemia in diabetic population.

The increased glucose for the long period of time is one of the stimulus factor for malignancy. ${ }^{4}$ The insulin resistance and the deranged lipid profile additionally increase the risk. Thus, increased ceruloplasmin level in diabetic group may indicate the risk of malignancy. Further the study has shown that natural killer cells have a significantly higher ceruloplasmin expression compared to all other major lymphocyte subsets. ${ }^{12}$ Moreover, ceruloplasmin is a novel adipokine with increased expression in adipose tissue of obese subjects as well as in cells from obesity-associated cancers. ${ }^{13}$ Adipokines, adiponectin and leptin, have in been linked to the development of malignant tumors through their action on cognate receptors affecting insulin sensitivity and activating cancer-associated signaling. ${ }^{13}$ Thus, increased level of ceruloplasmin could be taken as ominous sign of malignancy risk, as ceruloplasmin along with adiponectin and leptin are raised in the diabetic patients.

This study has revealed the correlation of ceruloplasmin with lipid ratio. The importance of lipid ratio has been verified with respect to cardio vascular status in many studies. Study has also highlighted the risk of malignancy in type II diabetes mellitus if serum ceruloplasmin is very high. The limitation of this study was that the dietary pattern was not considered. Similarly, prospective longitudinal studies are required to further elucidate the relationship between ceruloplasmin as a potential marker of glycemic status and its relationship with lipid profile which reflects the probable risk of cardiovascular problem in type II diabetes mellitus patients.

\section{CONCLUSION}

Serum ceruloplasmin could be used for evaluating the overall glycemic and cardiovascular status in patients with type II diabetes mellitus.

\section{ACKNOWLEDGEMENT}

Authors would like to acknowledge laboratory staffs Mrs Rushila Chitrakar, Mrs Samita Bajracharya and Ms Palpasa Maharjan for their technical assistance. 


\section{CONFLICTS OF INTEREST}

All authors declare no conflict of interest.

\section{REFERENCES}

1. Bambolkar S and Sainani GS. Evaluation of oxidative stress in diabetics with or without vascular complications. The Journal of the Association of Physicians of India 1995 Jan;43(1):10-12.

2. Robertson RP. Chronic oxidative stress as a central mechanism for glucose toxicity in pancreatic islet beta cells in diabetes. Journal of Biological Chemistry 2004;279(41):42351-42354.

3. Zozulinska D and Wierusz-Wysocka B. Type 2 diabetes mellitus as inflammatory disease. Diabetes Research and Clinical Practice 2006;74(2): S12-S16.

4. Ryu TY, Park J and Scherer PE. Hyperglycemia as a risk factor for cancer progression. Diabetes \& metabolism journal 2014;38(5):330-336.

5. Lee MJ, Jung CH, Kang YM, Jang JE, Leem J, Park JY, et al. Serum Ceruloplasmin Level as a Predictor for the Progression of Diabetic Nephropathy in Korean Men with Type 2 Diabetes Mellitus. Diabetes \& metabolism journal 2015;39(3):230-239.

6. Shukla N, Maher J, Masters J, Angelini GD and Jeremy JY. Does oxidative stress change ceruloplasmin from a protective to a vasculopathic factor? Atherosclerosis 2006;187(2):238-250.
7. Varela AS, Saez JB and Senra DQ. Serum ceruloplasmin as a diagnostic marker of cancer. Cancer letters 1997; 121(2):139-145.

8. Nasif ZN. Evaluation of Ceruloplasmin Oxidase Activity and C-Reactive Protein in the Sera of Patients with Diabetes Mellitus. National Journal of Chemistry 2010; 37: 175-185.

9. Enomoto $M$, Adachi $H$, Hirai $Y$, Fukami A, Satoh A, Otsuka $\mathrm{M}$, et al. LDL-C/HDL-C Ratio Predicts Carotid Intima-Media Thickness Progression Better Than HDL-C or LDL-C Alone. J Lipids 2011;2011(1):1-6.

10. Fox PL, Mazumder B, Ehrenwald E and Mukhopadhyay CK. Ceruloplasmin and cardiovascular disease. Free Radical Biology and Medicine 2000;28(12):1735-1744.

11. Lemieux I, Lamarche B, Couillard C, Pascot A, Cantin B, Bergeron J, et al. Total cholesterol/HDL cholesterol ratio vs LDL cholesterol/HDL cholesterol ratio as indices of ischemic heart disease risk in men: the Quebec Cardiovascular Study. Archives of internal medicine 2001; 161(22):2685-2692.

12. Banha J, Marques L, Oliveira R, de Fátima Martins $M$, Paixão E, et al. Ceruloplasmin expression by human peripheral blood lymphocytes: a new link between immunity and iron metabolism. Free Radical Biology and Medicine 2008; 44(3):483-492.

13. Arner E, Forrest AR, Ehrlund A, Mejhert N, Itoh M, Kawaji H, et al. Ceruloplasmin is a novel adipokine which is overexpressed in adipose tissue of obese subjects and in obesity-associated cancer cells. PloS one 2014 Mar 27;9(3): e80274.

\section{Authors Contribution:}

AT and VP- Designed the study; BA and SS- Performed the laboratory test; BKY, ETT, AB and MR- Revised the manuscript; VKS- Final approval.

\section{Orcid ID:}

Dr. Vijay Kumar Sharma - (i) https://orcid.org/0000-0002-9092-4957

Dr. Arjun Tumbapo - (1) https://orcid.org/0000-0001-8002-726X

Dr. Vivek Pant - (D) https://orcid.org/0000-0002-3967-1851

Mr. Binod Aryal - (1) https://orcid.org/0000-0003-4328-1212

Ms Sweta Shrestha - io https://orcid.org/0000-0002-4841-4603

Dr. Binod Kumar Yadav - (1) https://orcid.org/0000-0002-2839-2769

Dr. Eans Tara Tuladhar - (D) https://orcid org/0000-0001-5705-3953

Dr. Aseem Bhattarai - (i) https://orcid.org/0000-0002-5393-0629

Mr.Mithileshwor Raut - id https://orcid.org/0000-0002-2235-9095

Source of Support: Nil, Conflict of Interest: None declared. 